\title{
Landwirtschaftliches Zentralblatt
}

\author{
ABTEILUNG IV \\ VETERINÄRMEDIZIN
}

8. JAH RGANG

1.963

AKA DEMIE-VERLAG - BERLIN

LZ. IV VETERINĀRMEDIZIN - JG. 1963 - NR. 2 - SEITE 225-448 - BERLIN - FEBRUAR 1963 


\section{LANDWIRTSCHAFTLICHES ZENTRALBLATT}

\section{KURATORIUM}

Prof. Dr. Gustav Becker - Quedlinburg

Prof. Dr. Johannes Dobberstein - Berlin

Prof. Dr. Ernst Ehwald - Eberswalde

Prof. Dr.-Ing. Heinrich Heyde - Berlin

Prof. Dr. Hermann Meusel - Halle/Saale

Prof. Dr. Kurt Nehring - Rośtock

Prof. Dr. Erwin Plachy - Berlin

Prof. Dr. Johannes Reinhold - Berlin

Prof. Dr. Heinz Rðhrer - Insel Riems bei Greifswald

Prof. Dr. Otto Rosenkranz - Böhlitz-Ehrenberg

Prof. Dr. Rudolf Schick - Groß-Lüsewitz, Krs. Rostock

Prof. Dr. Karl Schmalfuß - HaHe/Saale

Prof. Dr. Wilhelm Stahl - Rostock

Prof. Dr. Hans Stubbe - Gatersleben, Bez. Halle

\section{CHEFREDAKTEUR}

Dr. Ursula Wittbrodt - Berlin

\section{STELLVERTRETENDER CHEFREDAKTEUR}

Dr. Werner Kleeberg - Berlin

\section{ABTEILUNG VETERINÄRMEDIZIN}

\section{REDAKTIONSAUSSCHUSS}

Kim Djonchi - Pjöngjang/Nordkorea

Prof. Dr. Johannes Dobberstein - Berlin

Prof. Dr. Gottlieb Flückiger - Bern

Prof. Dr. K. Iwanow - Sofia

Prof. Dr. Vincenc Jelínek $\dagger$ - Prag

Prof. Dr. Rezső Manninger - Budapest

Prof. Dr. Uveis Maskar - Istanbul

Prof. Dr. Maximilian Pflücke - Potsdam

Prof. Dr. Ilie Popovici - Bukarest

Prof. Dr. Gaston Ramon - Paris

Prof. Dr. Alexander Sotow - Moskau

Prof. Dr. Ushio Tanaka - Tokio

Prof. Dr. Rudolf Wetzel - Gießen

Prof. Dr. Vittorio Zavagli - Rom

\section{REDAKTION}

Oberveterinärrat Dr. Kurt Reinhardt - Kleinmachnow Prof. Dr. habil. Ekkehard Wiesner - Berlin 